\title{
Characterization of the Physical Properties of the Coconut Fiber Residue With a View to its Agroindustrial Use
}

\section{Caracterización de las Propiedades Físicas del Residuo de Fibra de Coco con Vista a su Aprovechamiento Agroindustrial}

VII International Congress of Science, Technology, Entrepreneurship and Innovation (SECTEI 2020)

Corresponding Author:

M. L. Vaca-Cardenas

maritza.vaca@espoch.edu.ec

Published: 26 August 2021

Production and Hosting by

Knowledge E

(c) M. L. Vaca-Cardenas et al. This article is distributed under the terms of the Creative Commons Attribution License, which permits unrestricted use and redistribution provided that the original author and source are credited.
G OPEN ACCESS

\section{L. Vaca-Cardenas ${ }^{1}$, M. González ${ }^{1}$, and P. Vaca ${ }^{2}$}

1Docente Facultad de Ciencias Pecuarias, Escuela Superior Politécnica de Chimborazo, Riobamba, Ecuador

${ }^{2}$ Dirección provincial de ministerio del ambiente Chimborazo, Riobamba, Ecuador

\section{Abstract}

Ecuador is a multi-climatic country with the capacity to host a large number of plant species; one example is the coconut (Cocos nucifera), an ancestral food of tropical areas and warm climates, which is considered as raw material for various industrial processes, such as obtaining fats, oils, candies, etc. However, the external part of the coconut constituted by the bast or mesocarp has not been properly exploited. Coconut bast, which has high fiber content (coir fiber), from which three main types can be distinguished: long and thin, coarse, and shorter type, can become an agroindustrial alternative due to its mechanical properties. These properties are as good or better than synthetic fibers or polyester. This research therefore aims to characterize the physical parameters that determine the functionality of coconut fiber, such as its length, softness, brightness, color, diameter, and its appearance or slenderness, which is the relationship between length and diameter. This research also contributes to solving the environmental problem of waste generated by coconut production, moving towards a sustainable economy from an environmental point of view. This work determines that coconut fiber is composed of cellulose and wood that has low heat conductivity and impact resistance, and that its resistance and durability make it an adequate raw material in several production sectors.

Keywords: coconut fiber, characterization, properties, agribusiness.

\section{Resumen}

El Ecuador es un país multi climático con la capacidad de alojar una gran cantidad de especies vegetales, un ejemplo de ello es el coco (Cocos nucifera) un alimento ancestral de zonas tropicales y climas cálidos, este es considerado como materia prima de diversos procesos industriales como obtención de grasas, aceites, confites, etc., sin embargo, la parte externa del coco constituida por la estopa o mesocarpio no ha sido correctamente explotada. La estopa del coco por su alto contenido de fibra (fibra bonote), de la cual se pueden distinguir tres tipos principales: una larga y fina, una tosca y una más corta, se convertir en una alternativa agroindustrial debido a sus propiedades físico - mecánicas que son tan buenas o mejores que fibras sintéticas o el poliéster. Esta investigación tuvo por objeto caracterizar los parámetros físicos que describan la funcionalidad de la fibra de coco como son su longitud, suavidad, brillo, color. Para lo cual se realizó un estadística descriptiva de medidas de tendencia central como la media, se analizaron un total de 10 muestras de fibra de coco tomadas al azar de 
los $15 \mathrm{Kg}$ de fibra obtenida de 50 cocos, teniéndose como resultado que para la suavidad el $80 \%$ de los participantes la ubican en el octavo lugar y el restante $20 \%$ en el noveno, lo que permite inferir que se tara de una fibra gruesa, rígida y áspera, para el brillo y luminosidad el $90 \%$ de los observadores determinaron que la fibra no posee esta característica. El 70\% de las panelistas delimitaron que el color característico de la fibra fue el café claro y el restante $30 \%$ la considera de color beige.

Palabras Clave: fibra de coco, caracterización, propiedades, agroindustria.

\section{Introducción}

La variedad Cocos nucifera $L$., es considerada una fruta tropical de aspecto redondeado cuyas dimensiones oscilan entre 20 a $30 \mathrm{~cm}$, pudiendo alcanzar pesos de hasta $2,5 \mathrm{~kg}$. Es un fruto tropical que presenta una forma redondeada, mide de $20-30 \mathrm{~cm}$ de largo y puede llegar a pesar hasta $2,5 \mathrm{~kg}$. El fruto del cocotero se compone de una capa externa o exocarpio que se presenta suave o lignificado, en el mesocarpio se tienen tejidos fibrosos duros y constituye el $35 \%$ del total del fruto, en la parte más interna se encuentra la porción conocida como endocarpio que por lo general rodea a la semilla, conocido de manera común como testa [1].

La fibra de coco es considerada como una de las más duras y de menor longitud comparándola con otras fibras vegetales. El diámetro medio de las fibras es $1 \mathrm{~mm}$ y en longitud llegan a medir entre 15 y $30 \mathrm{~cm}$. Las fibras obtenidas del coco están constituidas principalmente por lignina, celulosa y hemicelulosa, componentes que le otorgan las propiedades absorción y retención de agua; la composición química de las fibras de coco depende en gran parte del grado de madurez del fruto, variedad y lugar de cultivo. Un aspecto tecnológico de gran importancia corresponde a su resistencia a la tensión, por lo que tienen la función de ser tejido de soporte. Su excelente capacidad de estiramiento le confieren diversas aplicaciones en los campos de la construcción, química analítica y el tratamiento de efluentes industriales [2], y en la elaboración de medios hidropónicos debido a que es una fuente valiosa de minerales, sobretodo de Potasio $(\mathrm{K}+)$ y Cloro (Cl-) [3].

De acuerdo con lo mencionado por FAO, 'La fibra de coco se la obtiene de la cascara del coco, la cual se elabora para obtener distintos colores' [4], Según el Censo Nacional Agropecuario, las palmeras de esta fruta están en Esmeraldas, Manabí, Guayas y Loja. La provincia que mayor producción la tiene es Esmeraldas, con un número de hectáreas que alcanzan el 77,26\% del total nacional, seguida de Manabí, con el 18,72\% [5]. La provincia de Esmeraldas cuenta con la mayor producción anual de esta fruta produciendo alrededor de 4011 hectáreas en los cantones de San Lorenzo y Eloy Alfaro. 
La fibra de estopa de coco, también llamada fibra bonote, presenta 3 tipos principales: fibra esteras o hilo: larga y fina, fibra de cerda: más tosca, y fibra para colchones: fibra más corta [6]. Al realizar la comparación de la fibra de coco con otras fibras duras, se puede mencionar que es relativamente corta, sus células fibrosas miden alrededor de $1 \mathrm{~mm}$ de longitud, en vez de un promedio de $2,5 \mathrm{~mm}$ en el caso de células de sisal, y $4,9 \mathrm{~mm}$ en las de abacá; las células de bonote tienen un diámetro medio de 15 micras, y un haz de fibras puede tener de 30 a 300 o más células en su sección transversal total [7].

La actividad de producción de coco genera muchos residuos que afectan a las condiciones medio ambientales de todas las zonas de comercialización, debido a que la cascara del coco viene a ser una fibra difícil de degradar si no se genera un tratamiento, por lo cual las personas que comercializan este tipo de producto, tan solo se dedican en desechar la cascara en zonas aledañas como quebradas colindados en ríos, botaderos de basura, en calles o en cualquier sitio para deshacerse de este desecho [8].

La acumulación de los desechos que se generan por la producción de coco se convierte en un grave problema, ya que causan la proliferación de insectos, atraen roedores, perjudican la salud de la población y genera contaminación visual. La fibra de coco es una fibra que se obtiene de la cáscara de los cocos luego de pelarlos. La fibra de coco se extrae de la zona externa del fruto del coco conocida como cubierta, La fibra del coco llamada bonote, la cual está compuesta por tres tipos de fibras: Una larga y fina conocida como esteras, una tosca llamada fibra de cerda y una más corta denominada fibra para colchones. La fibra de coco, se extrae de la cáscara que se encuentra entre la capa dura externa llamada Epicarpio y la interna llamada endocarpio, miden hasta $35 \mathrm{~cm}$ de largo y un diámetro aproximado de 12 a 25 micras. Los pelos de coco se utilizan en diversas aplicaciones debido a sus características únicas y ecológicas. Su recolección no solo aporta ingresos económicos a decenas de familias.

La fibra de coco depende mucho de la variedad de y tipo de especie de la planta del cocotero, en el mundo existe 3 variedades principales de cocoteros, el cocotero gigante que es utilizado para la producción de aceites esta variedad de coco se da entre los 6 a 9 años, con unos 60 a 80 frutos por planta por año, el cocotero enano un coco de menor tamaño, dulce como fruto, se emplean fundamentalmente para la producción de bebidas envasadas, su producción es a los 3 años, permitiendo una cosecha de 120 a 150 frutos por planta por año y por ultimo tenemos el cocotero hibrido que es la mezcla de las 2 variedades anteriores, son frutos de tamaño mediano o grande, buen sabor producción es a los cuatro años, con unos 120 a 140 frutos por planta por año. El cocotero hibrido es la variedad más utilizada a nivel mundial por todas las características físicas si hablamos de la fibra del coco [9].

La fibra de coco se clasifica por el grado de la madurez del fruto, ya que mientras más maduro la fibra se tornará de color café claro y es más rígida debido al contenido 
de lignina en su estructura. En el caso de la fibra del fruto verde es más flexible de color blanco, pero esta es más susceptible a daño por microorganismos dado que contiene mayor humedad. La fibra de coco es la única fibra natural relativamente resistente a los daños provocados por el agua salada [7]. En la actualidad Mercedes-Benz, Ford, Mitsubishi y Mazda utilizan la fibra de coco en sus accesorios para aligerar partes internas de los automóviles. Otro producto que puede ser fácilmente reemplazado por la fibra de coco en procesos industriales es el amianto mejor conocido como asbesto el cual es considerado un contaminante ambiental cancerígeno, ya que la fibra de coco por sus características naturales, respira mejor que los productos petroquímicos, no es combustible, ofrece más intercambiabilidad [10].

Es importante mencionar que las fibras de coco tienen alta resistencia natural contra los hongos y ácaros lo que las convierte en ideales para ser utilizadas en el proceso de fabricación de colchones, en el Ecuador tiene todo el potencial para desarrollar una industria basándose en la producción del coco, ya que por su ubicación geográfica cuenta con suelos adecuados para el desarrollo de cultivos de gran rendimiento y calidad. Entre las fibras vegetales, esta tiene una de las más altas concentraciones de lignina, que lo hace más fuerte pero menos flexible que el algodón e inadecuado para teñir. La Fibra de coco constituye un excelente sustrato, por su buena capacidad de retención de humedad, ofreciendo grandes ventajas para la mezcla con otros sustratos [11].

La fibra de coco posee la propiedad de estirarse más allá de su límite elástico sin romperse, de igual manera posee una capacidad de absorción permanente; su resistencia a la tracción es baja en comparación con la de abacá, y esta propiedad disminuye más por la inmersión en agua. Después de una inmersión de 4 meses la fibra de coco pierde de un 35 a 45\% de su resistencia [12]. En esta investigación se establece la capacidad de resistencia de la fibra que depende del grado en que los esfuerzos pueden serle transferidos desde la matriz, grado que a su vez está regido por las características intrínsecas de la fibra, como: resistencia a la tensión [13].

Según datos del Banco Central Del Ecuador los principales mercados ha donde está dirigido la comercialización del coco es a: España, Antillas Holandesas y Estados Unidos Es indiscutible mencionar que el coco es un producto muy demandado por el mercado nacional e internacional [14]. La fibra de coco es un tema donde se debe aprovechar las ventajas de tipo ecológico que dispone el país para la producción y comercialización de fibra de coco, por lo que se considera su ejecución conveniente en el aspecto social y económico, ya que se incrementaría la oferta de trabajo rural y se lograría disponer de materia prima para nuevos productos para la industria.

Los problemas en la economía ecuatoriana identificados a partir del año 2012 , derivaron en una reestructuración de la Matriz Productiva cuyo objetivo fue impulsar la 
Transformación de la Matriz productivo a través de diversificación de la producción agrícola nacional con la finalidad de plantear alterativas de materias primas ecoeficientes y que posean un valor agregado; de esta manera se fortalecerá la economía nacional. Considerando este análisis, la presente investigación pretende aportar con el estudio y propuesta de utilización de uno de los subproductos agroindustriales menos estudiado como lo es la fibra de coco.

\section{Metodología}

\subsection{Materiales}

- 50 cocos

- 2 sacos de plástico

- 1 martillo

- 2 cartones Maizena

- Moldes

- Pinzas

- Tijeras

- Equipos

- Balanza Elastómetro

\subsection{Instalaciones}

Laboratorio de fibras de la Facultad de Ciencias Pecuarias.

\subsubsection{Obtención del Mesocarpio de coco}

El mesocarpio de coco como materia prima se obtuvo en la ciudad de Ambato, en los mercados populares de dicha ciudad, estos desechos sólidos derivados del coco eran apilados en el sector de Huachi Grande. Los frutos del cocotero vacíos fueron sometidos a un proceso de selección en el que se consideraba el tamaño del coco, mientras mayor tamaño, mejores rendimientos en la obtención de la fibra.

\section{Extracción de fibras}

En esta fase del proceso se inició con la obtención del mesocarpio a través de la acción mecánica (martillo). Luego de ablandar de forma mecánica al mesocarpio del fruto, se 
procedió a la separación de las fibras, en este proceso se utilizó una herramienta de corte, la misma que perfora la cáscara para removerla. Posteriormente en un recipie nte con agua, se sumerge el mesocarpio por un lapso de tiempo de $24 \mathrm{hr}$, protegidos de la luz y a temperatura ambiente, mientras ocurre el proceso de maceración. Este proceso es conocido como retting. Una vez que se logró desprender las fibras de los residuos del coco, se continúa con el proceso de lavado para eliminar impurezas en las fibras obtenidas.

De los 50 cocos recolectados se obtuvo alrededor de $15 \mathrm{~kg}$ de fibra total, rendimiento obtenido luego del proceso de lavado, siendo sometida a un secado natural que se realizó mediante tendido en el suelo y recibiendo $3 \mathrm{hr}$ de sol durante 5 días para que elimine la mayor cantidad de humedad que tenía la fibra. Cuando las fibras de coco estuvieron completamente secas y se notó que no tenga rastro de humedad se procedió a realizar cortes de la fibra y se le acomodaron en un cartón. Posteriormente como completo a las posibles formas de uso se procedió a realizar un engrudo a base de maicena permitiendo la adherirse de las fibras unas con otras.

Al mezclar el engrudo con la fibra de coco se formó una pasta espesa que se procedió a colocar en moldes para facilitar la forma de los maseteros que se realizaron. Cuando la pasta estuvo compacta en los moldes se procedió a realizar un secado, este secado duró alrededor de 2 días al sol para que se solidifique de forma correcta, posteriormente se retiró de los moldes obteniendo un masetero a base de fibra de coco que sirve para colocar plantas ornamentales. Cuando las masetas contuvieron a la planta, se procedió a regarlas estas no se destruyeron y manteniendo la humedad de la tierra por mayor tiempo.

\section{Evaluación de suavidad}

La técnica sensorial táctil es el método tradicional utilizado para evaluar subjetivamente los conceptos estéticos de la fibra, es decir básicamente la preferencia de la gente. Por lo tanto, las personas que utilizan los descriptores de los atributos mecánicosensoriales como suavidad, tersura, rigidez o dureza; sin embargo, puede haber algunas dificultades con este método de evaluación subjetivo.

\section{Escalas de los productos de referencia}

Se desarrollaron escalas estándar de clasificación de los atributos texturales con el objetivo de evaluar cuantitativamente los atributos mecánicos de textura con el fin de brindar un método de evaluación cuantitativo de los atributos mecánicos de textura. Esta escala se basa en los intervalos de intensidades de los atributos mecánicos encontrados. Las escalas descritas ofrecen una base para evaluación cuantitativa de 
textura y los valores resultantes dan un 'perfil de textura'. La metodología utilizada inicia con la presentación de la clasificación de atributos texturales. Se presentan al panel las definiciones de los atributos mecánicos. Los evaluadores estudian cada uno de los atributos mediante evaluaciones repetitivas de los productos de referencia seleccionados que representan puntos en las escalas de referencia. Se les pide clasificar los ejemplos dados de acuerdo con la escala valores de 1 a 10 [16].

\section{Caracterización del color de la fibra de coco}

El procedimiento para identificación del color de fibra de un tejido, depende de la naturaleza de la muestra. La metodología aplicada utiliza pruebas sencillas como la inspección visual, que se basa en el aspecto y tacto de la fibra [17]. En esta prueba se establecieron dos parámetros de clasificación Beige B y Café Claro CC, estos parámetros fueron aplicados al panel de evaluadores los cuales clasificaron las muestras presentadas de acuerdo a su percepción visual.

\section{Determinación de brillo y luminosidad de la fibra}

El brillo es una percepción visual como resultado de la evaluación de las superficies. Cuanta más luz directa se refleja, mayor percepción de brillo se obtiene, la medición de este parámetro en las fibras obtenidas del coco ha respetado la siguiente escala:

\section{\begin{tabular}{|ll|l|l|l|l|l|l|l|l|l|}
\hline & $10 \%$ & $20 \%$ & $30 \%$ & $40 \%$ & $50 \%$ & $60 \%$ & $70 \%$ & $80 \%$ & $90 \%$ & $100 \%$ \\
\hline
\end{tabular}}

Figure 1

Notación nominal descriptiva del brillo. Fuente: Diccionario Akal del color. Akal. Especificación [18].

\subsubsection{Medida de la longitud}

De manera inicial se eligieron fibras individuales al azar y se procedió a la medición de su longitud con una regla, con una precisión de $1 \mathrm{~mm}$. Para llevar a cabo la medición se colocó la fibra sobre una mesa de trabajo y se sujetó a los extremos de la fibra y proceder a la medición. Para mejorar la visibilidad de la medida se utilizó un lente de aumento (lupa). El análisis de la longitud se mide en centésimas de pulgada y en milímetros. Cuando se trata de fines comerciales, la longitud del instrumento suele convertirse en la longitud de fibra. En la Tabla 1 puede verse la conversión de pulgadas (32 avos de pulgada) en longitud de fibra. 


\section{Table 1}

Cuadro de conversión longitud de fibra upland.

\begin{tabular}{|l|l|}
\hline Pulgadas & 32 avos \\
\hline 0,79 y más & 24 \\
\hline $0,80-0,85$ & 26 \\
\hline $0,86-0,89$ & 28 \\
$0,90-0,92$ & 29 \\
$0,93-0,95$ & 30 \\
$0,96-0,98$ & 31 \\
$0,99-1,01$ & 32 \\
\hline $1,02-1,04$ & 33 \\
$1,05-1,07$ & 34 \\
\hline $1,08-1,10$ & 35 \\
\hline
\end{tabular}

Pulgadas
$1,11-1,13$
$1,14-1,17$
$1,18-1,20$
$1,21-1,23$
$1,24-1,26$
$1,27-1,29$
$1,30-1,32$
$1,33-1,35$
1,36 y más largas

32 avos
37
37
38
39
40
41
42
43
44 y más largas

Fuente: Guía del exportador de algodón [19]

\subsubsection{Medida de la resistencia}

Para la medida de la resistencia fue utilizada la misma muestra que para la medida de longitud de la fibra. En el proceso se utilizan pinzas con las cuales se sujetan separadas entre sí a una distancia de 1/8" y se mide la fuerza necesaria para romper las fibras. La resistencia se mide en gramos por tex (g/tex). La unidad tex equivale al peso en gramos de 1000 metros de fibra. Por consiguiente, la resistencia obtenida es la fuerza en gramos necesaria para romper un haz de fibras de 1 tex. Existe una relación directamente proporcional entre la resistencia de la fibra y la resistencia del hilo. La resistencia de la fibra depende de la variedad, contenido nutricional de la planta de donde proviene la fibra e incluso por las condiciones meteorológicas [19].

\section{Resultados y Discusión}

En la Tabla 2 se muestran los resultados correspondientes a la determinación de suavidad de la fibra. La medida de la suavidad se relaciona a la finura y diámetro de la fibra, el parámetro de suavidad es determinante para el comportamiento y sensación al tacto que presenten las fibras de origen animal o vegetal [1]. En el presente estudio se analizaron los resultados de la evaluación sensorial de la fibra de coco en una escala de 1 al 10, siendo 1 el grado de mayor suavidad y 10 el valor correspondiente a suavidad nula. De las 10 evaluaciones un $80 \%$ ubican a la fibra de coco en octavo lugar respecto a la suavidad, mientras que un $20 \%$ de los evaluadores colocaron a la fibra de coco en el noveno lugar de la escala. Estos resultados permiten determinar una suavidad casi nula de la fibra de coco. 
Table 2

Resultados obtenidos en la evaluación sensorial de suavidad y finura para la fibra de coco.

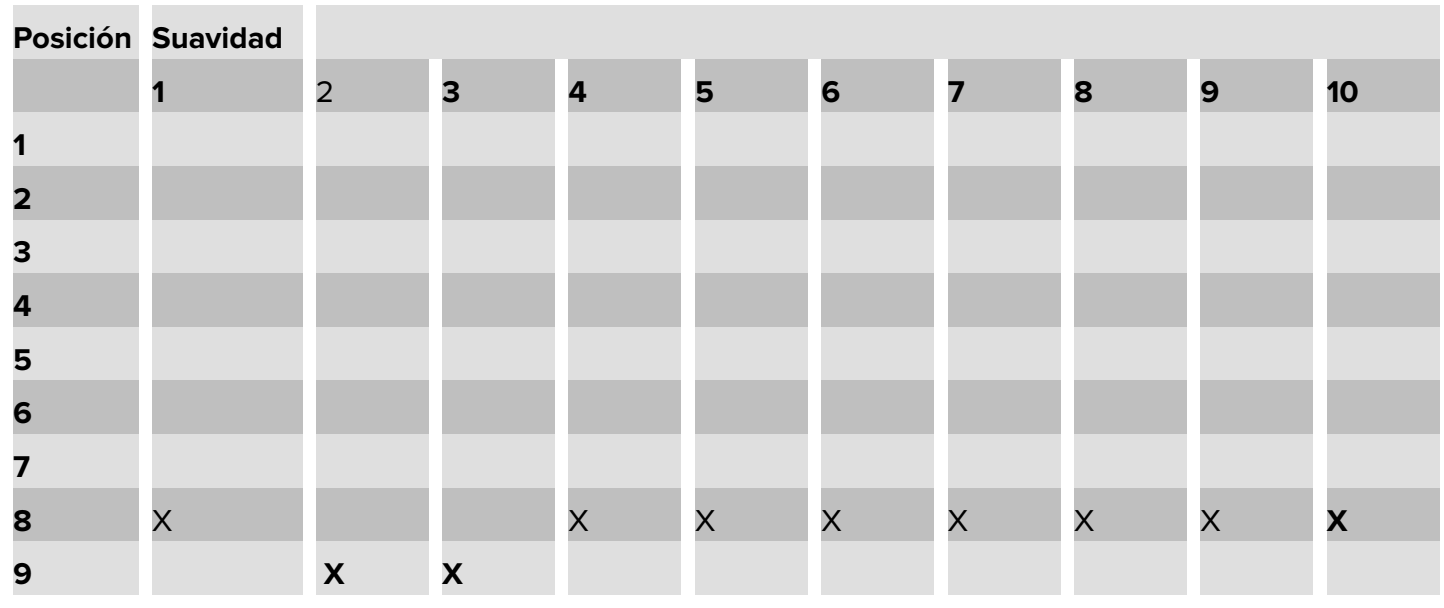

El proceso de evaluación sensorial de la suavidad de fibras, permite clasificar las fibras con respecto a la finura, esta clasificación establece fibras gruesas, rígidas y ásperas, con mayor firmeza y resistencia al arrugado; así mismo establece la categoría de fibras finas, suaves y flexibles [20]. Con respecto a esta clasificación se puede clasificar a la fibra de coco analizada en el presente estudio como una fibra gruesa. La suavidad es un aspecto que influye durante el proceso textil en el proceso de hilatura, regularidad de los hilos, distribución de fibras en la mezcla, brillo de hilos y tejidos, absorción del colorante, dependiendo de la finura establece intensidades diferentes.

Los datos presentados en la Tabla 3 corresponden a la evaluación sensorial del Brillo para la fibra de coco. Desde el punto de vista visual, se puede mencionar que cada material posee características intrínsecas que se traducen en una sensación luminosa y cromática que se manifiesta en aspectos como tonalidad, claridad, luminosidad o saturación. Dependiendo de las características visuales de un material específico, estás pueden ser aprovechadas para atribuir cualidades sensoriales útiles y comunicativas [21].

Table 3

Resultados de la evaluación sensorial brillo y luminosidad para la fibra de coco.

\begin{tabular}{l|l|l|l} 
Brillo/Luminosidad & \multicolumn{2}{l}{ Observación } & Porcentaje \\
\hline Si & Puntuació & 1 & $10 \%$ \\
No & 1 & 9 & $90 \%$ \\
& 0 & 10 & $100 \%$
\end{tabular}

De acuerdo con los resultados de la evaluación sensorial realizada en esta investigación (Tabla 3), se estableció que la fibra de coco no posee características visuales 
como la luminosidad y el brillo. Tanto las fibras duras como blandas permiten con su tejido visualizar diferentes texturas relativas a la luminosidad relacionada con la forma en que la luz interactúa con la superficie del producto. En la investigación planteada se usó el término opacidad para describir las propiedades visuales de la fibra de coco. Esta condición de opacidad en las fibras de coco estudiadas, están relacionadas con la humedad superficial y viveza del color que son los determinantes para el brillo o bien la opacidad.

Otro de los aspectos analizados corresponde al color en donde se establecieron dos variedades, Beige B y Café Claro CC, lo resultados se muestran en la Tabla 4. En las fibras evaluadas el $30 \%$ de jueces escogieron al color beige $\mathrm{B}$, mientras que el $70 \%$ selecciono la variedad color café claro CC.

\section{Table 4}

Resultados de la evaluación sensorial parámetro color para la fibra de coco.

\begin{tabular}{l|l|l|l}
\hline Color & \multicolumn{1}{l}{ Suma } & Porcentaje \\
\hline Color & Codigo & 3 & $30 \%$ \\
\hline Beige & B & 7 & $70 \%$ \\
\hline Café claro & CC & 10 & $100 \%$
\end{tabular}

Los métodos de obtención, maduración y desarrollo de la fruta pueden establecer dos tonalidades: Fibras color marrón obtenida de cocos ya maduros, presentan mayor grosor y resistencia a la abrasión, son menos flexibles y forman hilos enroscados. La otra tonalidad posible corresponde a un color claro, son fibras extraídas antes de madurar, más finas, lisas y por ello más débiles [22].

Una fibra puede ser caracterizada a través de parámetros como longitud, diámetro y aspecto o esbeltez. Según su origen, las fibras pueden ser clasificadas en sintéticas y naturales de estos dos grandes grupos se tienen diversas formas: redondas, rectangulares, dentadas, rizadas, con extremos en gancho, en malla o agrupadas por encolamiento. La fibra de coco puede clasificarse en 3 tipos tomando en consideración la longitud de sus fibras: una más larga y fina que se conoce con el nombre de fibra de esteras o hilo; una más tosca, que se conoce con el nombre de fibra de cerda, y una fibra más corta, conocida con el nombre de fibra para colchones [23].

\section{Table 5}

Total de Medidas de longitud de la fibra de coco.

\begin{tabular}{|c|c|c|c|c|c|c|c|c|c|c|c|}
\hline \multicolumn{12}{|c|}{ Longitud (cm) } \\
\hline \multicolumn{12}{|c|}{ Observaciones } \\
\hline 1 & 2 & 3 & 4 & 5 & 6 & 7 & 8 & 9 & 10 & $\sum x^{*} t$ & $\dot{x}$ \\
\hline 10 & 15.5 & 16 & 11 & 16 & 15 & 15,5 & 15,6 & 13,8 & 24,1 & 152, & 15,25 \\
\hline
\end{tabular}


En comparación con otras fibras duras de origen vegetal la del coco es relativamente corta con un haz de fibras que puede tener de 30 a 300 o más células en su sección transversal total. La fibra cortada del coco varía entre 15 y $35 \mathrm{~cm}$. Su diámetro varía de 0,1-1,5 mm frente a 0,2-1 mm que tiene el abacá.

La fibra de bonote tiene una capacidad de estiramiento de 29,04\%, con un módulo de rigidez de 1.8924 dinas $/ \mathrm{cm}^{2}$ [24]. La presente investigación establece un análisis estadístico de acuerdo a las medidas tomadas de la fibra (Tabla 5), la longitud de la fibra de coco no varía, al contrario, presentan las mismas medidas por lo cual ayuda a la compactación, la dificultad más notable es el tamaño reducido de cada fibra.

La resistencia es un aspecto de gran importancia en el análisis de las características físicas y mecánicas de las fibras. Con respecto a la fibra del coco es importante considerar las propiedades que tiene de estirarse más allá de su límite elástico sin romperse, así como su capacidad de absorber un estiramiento permanente cuando soporta esta carga; aunque la resistencia a la tracción de la fibra de coco seca es baja en comparación con la de otras fibras de origen vegetal, disminuye mucho menos por inmersión en agua. Después de 4 meses de inmersión, la fibra de coco pierde solamente $35-45 \%$ de su resistencia.

El porcentaje de rendimiento de las fibras analizadas, dependen del tamaño de los cocos, madurez, variedad y métodos de extracción aplicados. En la Tabla 6 se muestran las medidas obtenidas del ensayo de resistencia para las fibras de coco se han realizado 10 ensayos en total y luego se ha procedido a realizar el respectivo análisis estadístico descriptivo correspondiente a las medidas de tendencia central como es la media.

\section{Table 6}

Medidas de resistencia para fibras de coco.

\section{Resistencia (Newtons N)}

Observaciones

\begin{tabular}{l|l|l|l|l|l|l|l|l|l|l|l|}
1 & 2 & 3 & 4 & 5 & 6 & 7 & 8 & 9 & 10 & $\sum \mathrm{X}^{*} \dagger$ & $\dot{x}$ \\
27 & 30,65 & 32 & 30 & 42,3 & 43,5 & 45 & 39,8 & 38,8 & 40 & 369,05 & 36,905
\end{tabular}

\section{Conclusiones}

De acuerdo a los resultados obtenidos de la presente investigación, se ha llegado a concluir que:

- Con el análisis respectivo se determinó que para la suavidad de la fibra de coco el $80 \%$ de los participantes la ubican en el octavo lugar y el restante $20 \%$ en el noveno, lo que permite inferir que se tara de una fibra gruesa, rígida y áspera. 
- Para el brillo y luminosidad el $90 \%$ de los observadores determinaron que la fibra no posee esta característica. El 70\% de las panelistas delimitaron que el color que presento la fibra de bonete es café claro y el 30\% la considera de color beige.

- En cuanto a la longitud de la fibra de coco se pudo determinar que se trata de una fibra corta ya que su media fue de $15,25 \mathrm{~cm}$. Para la variable resistencia se pudo comprobar que tiene una media de $36.0905 \mathrm{~N}$.

\section{Anexos}

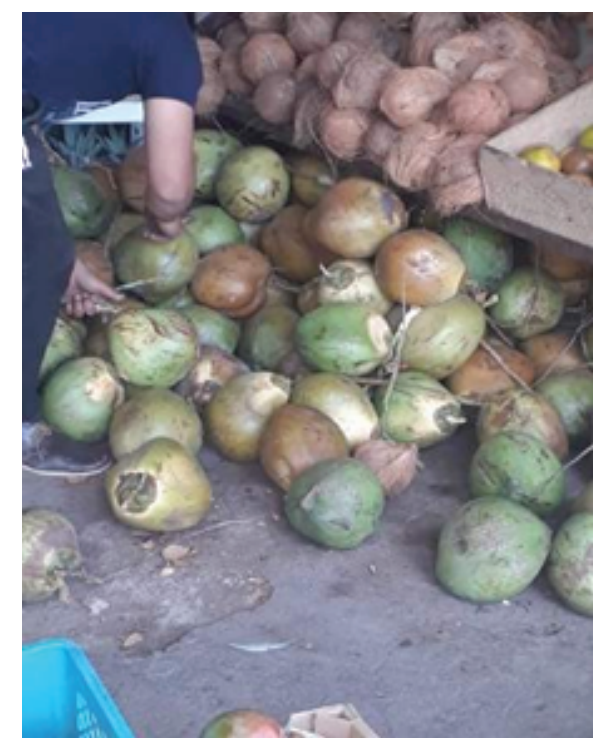

Figure 2

Selección de los cocos.

\section{References}

[1] León, J. Botánica de los cultivos tropicales. San José:IICA;1987.

[2] Anirudhan T, Unnithan, M. Arsenic (V) removal from aqueous solutions using an anion exchanger derived from coconut coir pith and its recovery. Chemosphere. 2007;60-66.

[3] Yahya A, Safie H, Kahar S. Properties of cocopeat-based growing media and their effects on two annual ornamentals. Journal of Tropical and Agriculture Food Science. 1997;25:151- 157.

[4] FAO. Materias primas agrícolas. Roma: FAO; 2010.

[5] Velasco, B. Esmeraldas concentra la palma de coco. Revista Líderes;2017. 


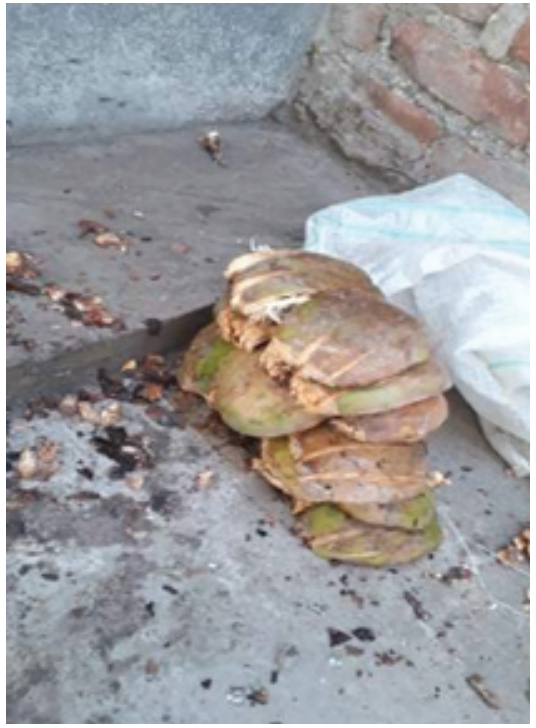

\section{Figure 3}

Prensado de la cáscara del coco.

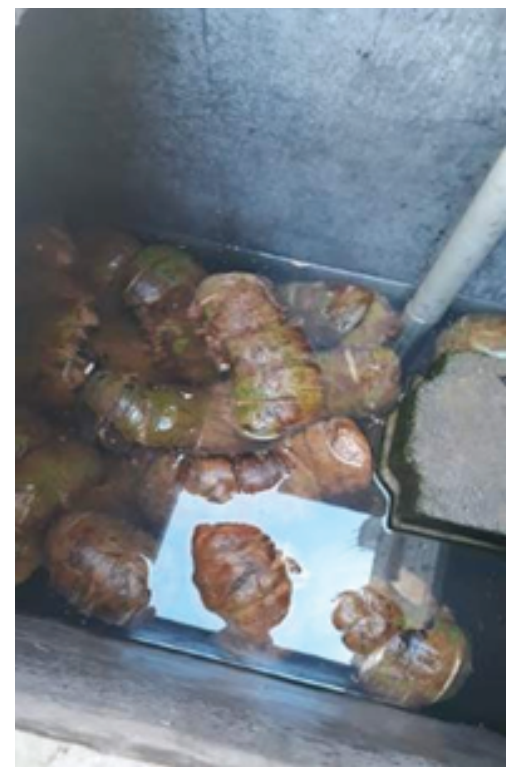

\section{Figure 4}

Remojo del coco para la.

[6] Cavides H, Rojas W. Evaluación de la estopa de coco como fuente alternativa de fibra celulósica para papel. Tesis (Ingeniero Químico). Santiago de Cali:Universidad del Valle;1994.

[7] Freire, L. (2012). Esmeraldas digital noticias. Available from http://esmeraldas2012. blogspot.com/

[8] QUINTERO G, S.L. (2003) Evaluación del uso de la estopa de coco (Cocos nucifera), obtenida como residuo de la industria alimenticia en el Valle del Cauca, para la 


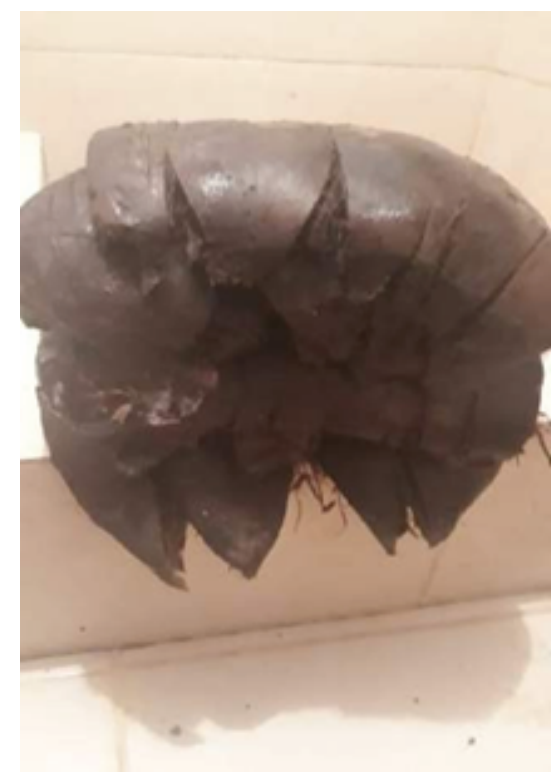

\section{Figure 5}

Escurrido del coco obtención de fibra.

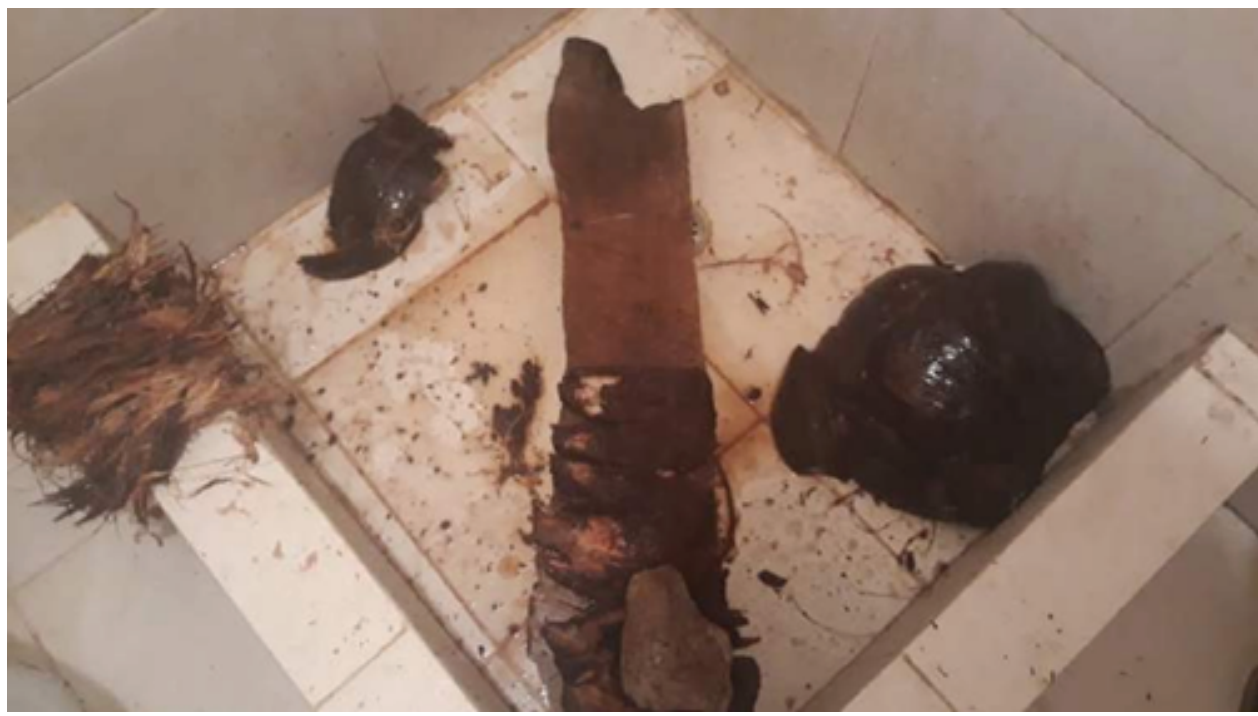

Figure 6

Obtención de la fibra.

elaboración de elementos prefabricados de concreto aligerado [Trabajo de grado Ingeniero Agroindustrial]. Palmira: Universidad Nacional de Colombia, Sede Palmira, Facultad de Ingeniería y Administración; 2003.

[9] Granados D, López GF. Manejo de la palma de coco. México;2015.

[10] Gallegos, S. Obtención de un material compuesto de matriz elastomérica y fibra de coco. Quito: EPN; 2011. 


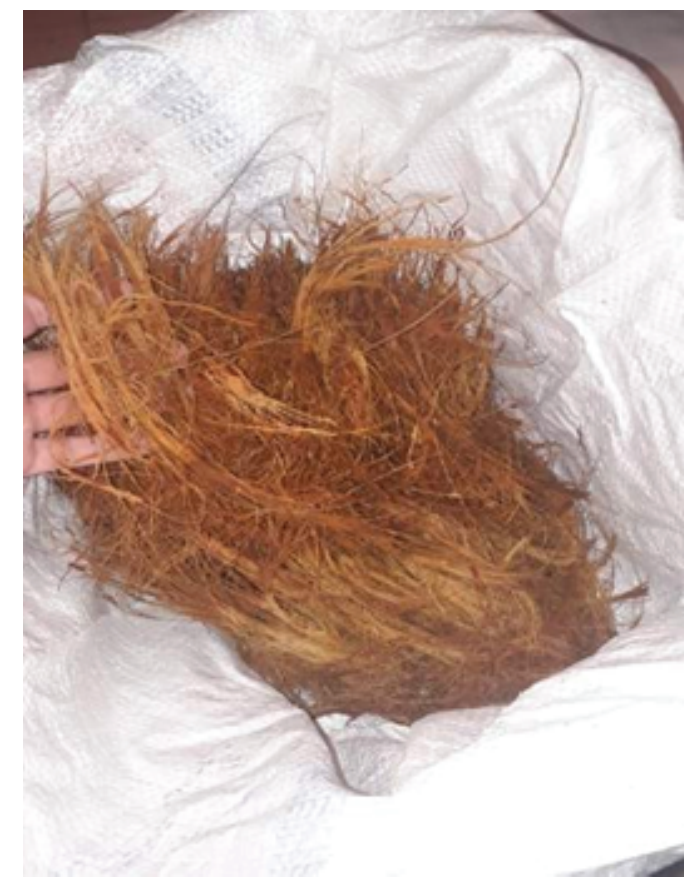

Figure 7

Secado de la fibra.

[11] Montañez AL. Utilización de la fibra de coco como sustituto del Amianto en los procesos industriales. Revista de Ingeniería, 2009; 20-26.

[12] FAO. (2014). Fibras del futuro. Obtenido de Fibras del futuro. Rome: FAO; 2014. Available from http://www.fao.org

[13] Grimwood, EB. Los productos del cocotero. Colección FAO: Producción y protección vegetal. Rome: FAO; 1977.

[14] Sandoval E, Bocanegra RA. Análisis del comportamiento mecánico del concreto fibro reforzado [Thesis in Ingeniero Civil]. Santiago de Cali: Universidad del Valle; 1997.

[15] Banco Central del Ecuador. Estadísticas ecuatorianas. Obtenido de Banco Central del Ecuador; 2015.

[16] ICONTEC. NTC 4489, Análisis Sensorial. Metodología. Perfil de Textura. Bogotá; 2016.

[17] INSTITUTO HOLLEN. Introducción a los textiles. México: Ed Limusa; 2002.

[18] Gallego R, Sanz J. Diccionario Akal del color. Akal. 2001; pp 365.

[19] Centro De Comercio Internacional UNCTAD/OMC Guía del Exportador de Algodón. Ginebra: CCl; 2007.

[20] Carpenter R, Lyon D, Hasdell T. Análisis sensorial en el desarrollo y control de la calidad de alimentos. Editorial Acribia. Segunda edición. Zaragoza; 2000.

[21] Alonso, J. Manual control de calidad en productos textiles y afines. Madrid; 2015. 
[22] Futonia. (2015). Colchón Fibra de coco. Futonia. 2015. Available from: http://www. futonia.com/futones-ycolchones/colchon-fibra-de-coco-37.

[23] Cavides H, Rojas W. Evaluación de la estopa de coco como fuente alternativa de fibra celulósica para papel [Thesis in Ingeniero Químico]. Santiago de Cali: Universidad del Valle, 1994.

[24] Quintero G, SL. (2003). Evaluación del uso de la estopa de coco (Cocos nucifera), obtenida como residuo de la industria alimenticia en el Valle del Cauca, para la elaboración de elementos prefabricados de concreto aligerado [Trabajo de grado Ingeniero Agroindustrial]. Palmira: Universidad Nacional de Colombia, Sede Palmira, Facultad de Ingeniería y Administración; 2003.

[25] Sandoval E, Bocanegra, RA. (1997). Análisis del comportamiento mecánico del concreto fibro reforzado [Tesis Ingeniero Civil]. Santiago de Cali: Universidad del Valle; 1997. 